\title{
La médecine de reproduction en Suisse
}

\author{
Rahel Gürbera, Nikolaus Zwicky-Aeberhard ${ }^{\text {b }}$ \\ Pour la "Vereinigung katholischer Ärzte der Schweiz (VKAS)» \\ ${ }^{a}$ Dr, présidente VKAS; ${ }^{b}$ Dr, président sortant VKAS
}

Dimanche, 14 juin 2015 le peuple suisse vote si la Constitution fédérale devait être modifiée de manière que le diagnostic de préimplantation (DPI) pouvait être utilisé dès lors en Suisse.

\section{Réflexions sur le status de l'embryon humain}

Celles-ci sont la base à une évaluation éthique du DPI. Que celui-ci est accepté ou refusé dépend du fait si la dignité et l'état de personne de l'homme dès la fécondation présente un processus par étapes, donc graduel, ou si, dès l'union d'une ovule et d'un spermatocyte, l'homme est à considérer comme une personne avec une dignité de protection complète. Longtemps avant des connaissances biologiques du status de l’embryon humain Immanuel Kant dans sa Metaphysik der Sitten a justifié qu'avec l'union d'un ovocyte et un spermatocyte un nouvel individu humain est à naître, doté d'un génome propre, a ainsi justifié philosophiquement la détermination du Landrecht prussien selon lequel les «droits de l'humanité» sont valables également aux enfants à naître dès le moment de leur conception [1]. Donc pas d'échelonnement selon le stade de

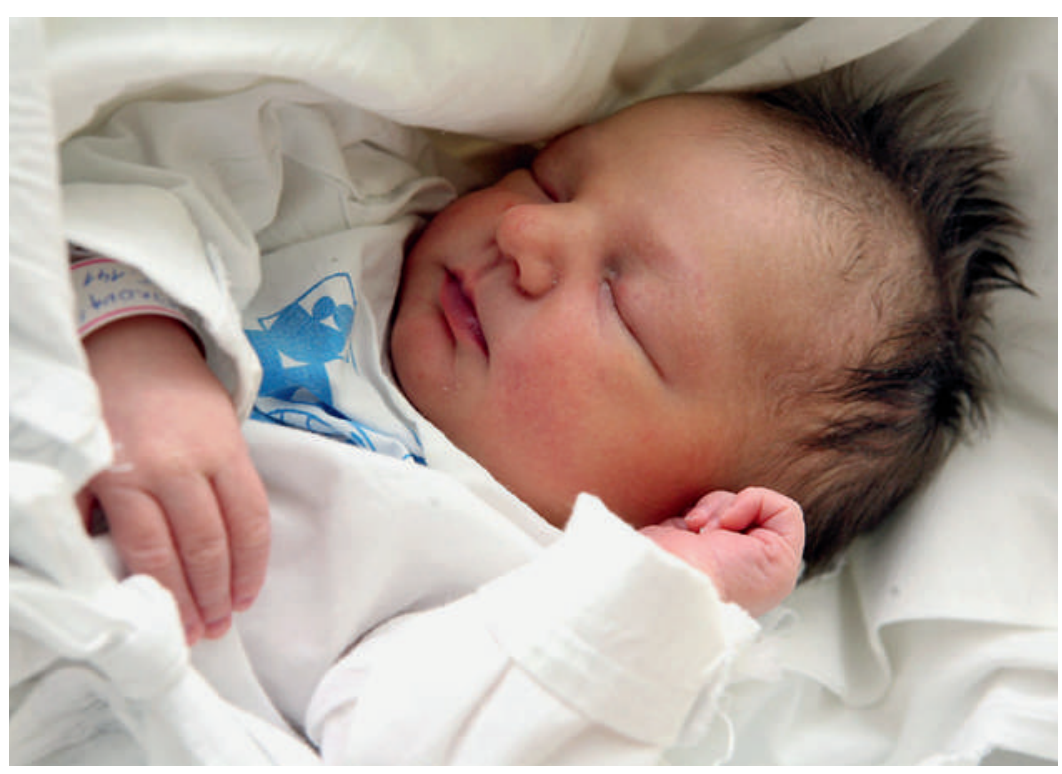

Un enfant c'est un cadeau. développement. Quant à la vie des embryons humains ceci veut dire que également dans leur phase précoce de leur existence ils ne peuvent être soumis à une délibération de biens. Quant au DPI: un contrôle de qualité, et c'est ça le DPI, va à l'encontre de la dignité humaine et est à considérer comme l'expression d'une sélection eugénique, c-à.-d. d'une prétention de décider, si une vie est digne de vivre ou non.

\section{Concernant la modification de la Constitu- tion et la modification consécutive de la loi}

Selon la Loi fédérale sur la procréation médicalement assistée (LPMA), valable depuis 2001, il est interdit de séparer une ou plusieurs cellules d'un embryon et aussi de l'examiner. La législation voulait ainsi empêcher le DPI. Dans son message le Conseil fédéral écrivait en 1996 textuellement:

«Si le DPI devient le standard de l'art médical le seuil qui freine pourrait baisser progressivement afin d'examiner l'embryon selon des critères quelconques et d'éclaircir des caractères désirés avant le transfer. La fixation d'une limite entre prévention admise et une sélection ne sera ainsi plus possible» [2]. Il est étonnant que ce pronostic très réaliste de l'ancien Conseil fédéral ne trouve plus d'attention au bout des 20 ans. Mémoire à court terme utilitariste des politiciens?

Toute la médecine de reproduction est dominée d'une mentalité utilitariste qui ne voit que le pas suivant «utile» et il le justifie par le succès de la recherche. La législation boite d'après les faits et se lie à la force normatrice des faits.

Selon Art. 119 de la Constitution fédérale était valable: «... ne peuvent être développé qu'autant d'ovocytes humains à l'extérieur du corps de la femme vers des embryons que lui peuvent être implantés de suite». Ce passage devrait être modifié de façon suivante: «... ne peuvent être développé autant d'ovocytes humains à l'extérieur du corps de la femme vers d'embryons que sont nécessaires pour la reproduction médicalement assistée». Ici il s'agit d'un saut quantique, même si dans le projet pour la modification de la Loi fédérale sur la procréation médicalement assistée une limite supérieure 
arbitraire de 12 embryons est mentionnée. Pour ceci il en faut au moins une vingtaine, ce qui comporte une hyperstimulation ovarienne, compromettant la femme en question et mettant sa vie en danger. Le Primum nil nocere est un des commandements du médecin!

\section{DPI et diagnostic prénatal (DPN)}

Si le DPI est une méthode purement sélectionnante au désavantage de l'embryon «malade»: le DPN peut engendrer un traitement curatif intrauterin en cas d'embryon malade et ne signifie pas un avortement dans chaque cas. C'est aussi la raison pour laquelle nous donnerons un avis plutôt favorable au DPN pour autant qu'il est dans l'intérêt de l'embryon. Que le DPI serait une alternative possible à un examen par DPN pendant une grossesse est inexacte; après un DPI effectué on ne renoncerait pas par principe à un DPN (avec la conséquence possible d'un avortement).

Il est important à considérer le fait que le DPI amène le plus souvent à plusieurs embryons sains surnuméraires qui, si cryoconservés, seront transmis à la re-

\section{Un contrôle de qualité, et c'est ça le DPI,} va à l'encontre de la dignité humaine et est à considérer comme l'expression d'une sélection eugénique.

cherche ou détruits. La méthodique du DPI est en soi embryo-consumeur et amène nécessairement à la destruction de vie humaine: un prix élevé qui doit être payé pour empêcher une jeunesse malade génétiquement. danger est que ces personnes se fassent des reproches telles que l'on ait pu les empêcher à naître au lieu «d'être à la charge des Soins sociaux et de l'AI».

\section{Conclusion}

Avec l'introduction des techniques in vitro la limite à ne pas franchir, le Rubicon bio-éthique, fut franchi et ainsi les écluses pour une instrumentalisation des embryons humains. L'accès aux méthodes de fertilisation

\section{La méthodique du DPI est en soi embryo- consumeur et amène nécessairement à la destruction de vie humaine.}

extra-corporelles nous a amené le passage d'un enfant comme un cadeau attendu à un produit revendiqué [3]. Le DPI comporte une instrumentalisation subtile de l'homme, car «le DPI ne sert pas, comme le nom prétend de manière idéale au diagnostic, mais il est dirigé à priori vers la sélection de la vie humaine. Il est centré non seulement vers la faiblesse, mais vers les faibles, il est dirigé vers la destruction d'embryons et vers l'exclusion de principe de vie empêchée» [4].

\section{Références}

1 Kant I. Die Metaphysik der Sitten. §28 (AB112f) zit. bei Eberhard Schockenhoff in: Beckmann R, Löhr M (Hg.): Der Status des Embryos. Würzburg: Verlag Johann Wilhelm Naumann; 2003. S. 71

2 Schweizerischer Bundesrat, Botschaft über die Volksinitiative «Zum Schutz des Menschen vor Manipulationen in der Fortpflanzungstechnologie (Initiative für menschenwürdige Fortpflanzung FMF)" und zu einem Bundesgesetz über die medizinisch unterstützte Fortpflanzung (FMedG), 26. Juni 1996.

3 Di Pietro, ML, Giuli A, Serra A. La diagnosi preimpianto in Medicina e Morale. 2004(3):498. «Il ricorso alle techniche di fecondazione artificiale extracorporea ha portato alla trasformazione del figlio da dono atteso a prodotto preteso".

4 Koch K (damaliger Bischof von Basel, heute Kardinal in Rom). Grenzen der (Bio-)Medizin: Verfügbarkeit über das Leben? Vortrag an der Generalversammlung der Vereinigung katholischer Ärzte der Schweiz in Solothurn am 6. März 2004, abgedruckt in Acta Medica Catholica Helvetica 2/2004. 